\title{
Hořava Gravity is Asymptotically Free in $2+1$ Dimensions
}

\author{
Andrei O. Barvinsky, ${ }^{1,2}$ Diego Blas, ${ }^{3}$ Mario Herrero-Valea, ${ }^{4,}$ Sergey M. Sibiryakov, ${ }^{3,4,5}$ and Christian F. Steinwachs ${ }^{6}$ \\ ${ }^{1}$ Theory Department, Lebedev Physics Institute, Leninsky Prospect 53, Moscow 119991, Russia \\ ${ }^{2}$ Tomsk State University, Department of Physics, Lenin Ave. 36, Tomsk 634050, Russia \\ ${ }^{3}$ Theoretical Physics Department, CERN, CH-1211 Geneva 23, Switzerland \\ ${ }^{4}$ Institute of Physics, LPPC, Ecole Polytechnique Fédérale de Lausanne, CH-1015, Lausanne, Switzerland \\ ${ }^{5}$ Institute for Nuclear Research of the Russian Academy of Sciences, 60th October Anniversary Prospect, 7a, 117312 Moscow, Russia \\ ${ }^{6}$ Physikalisches Institut, Albert-Ludwigs-Universität Freiburg, Hermann-Herder-Strasse 3, 79104 Freiburg, Germany
}

(Received 21 July 2017; revised manuscript received 6 October 2017; published 20 November 2017)

\begin{abstract}
We compute the $\beta$ functions of marginal couplings in projectable Hořava gravity in $2+1$ spacetime dimensions. We show that the renormalization group flow has an asymptotically free fixed point in the ultraviolet (UV), establishing the theory as a UV-complete model with dynamical gravitational degrees of freedom. Therefore, this theory may serve as a toy model to study fundamental aspects of quantum gravity. Our results represent a step forward towards understanding the UV properties of realistic versions of Hořava gravity.
\end{abstract}

DOI: 10.1103/PhysRevLett.119.211301

Introduction.-Formulating a quantum theory of the gravitational interactions remains one of the major challenges in theoretical physics. Despite the impressive achievements in this direction, in particular of string theory, many fundamental questions still remain open. It is of particular interest whether such a theory can be formulated in the language of a unitary and perturbative quantum field theory in $3+1$ space-time dimensions, as is the case for all the other interactions in the standard model of particle physics (SM).

The main obstruction for this venture within general relativity (GR) is the well-known fact that the gravitational coupling constant, the Newton's constant $G$, is dimensionful for dimensions greater than $1+1$. This ultimately renders the theory perturbatively nonrenormalizable - with an increasing number of divergences appearing at every order in the loop expansion. A possible solution is to extend the gravitational Lagrangian by terms quadratic in the curvature, thereby increasing the number of derivatives acting on the metric field. Although this can make the theory renormalizable [1] and even asymptotically free $[2,3]$, it jeopardizes the unitarity of the theory.

This idea was revisited by Hořava, who suggested that unitarity and perturbative renormalizability could be reconciled in theories of gravity without Lorentz invariance (LI) [4]. More concretely, he suggested that if the universe is endowed with a preferred foliation into space and time, one can construct a power-counting renormalizable theory containing only marginal and relevant operators with respect to a Lifshitz (anisotropic) scaling

Published by the American Physical Society under the terms of the Creative Commons Attribution 4.0 International license. Further distribution of this work must maintain attribution to the author(s) and the published article's title, journal citation, and DOI.

$$
t \mapsto b^{-d} t, \quad x^{i} \mapsto b^{-1} x^{i},
$$

where $d$ is the number of spatial dimensions. This proposal requires a mechanism to account for the stringent tests of LI of the SM and some promising ideas have been suggested; see Ref. [5]. It is important to stress that so far "nonprojectable" Hořava gravity (defined below) is consistent with all phenomenological constraints [6,7]. Finally, apart from being a viable candidate for the ultraviolet (UV) completion of GR, Hořava gravity can be used as a gravitational dual for strongly coupled systems exhibiting the Lifshitz scaling $[8,9]$.

Several fundamental aspects of Hořava gravity must be clarified before declaring it a successful theory of quantum gravity. The first one is renormalizability beyond power counting. Recently, this has been proven for the projectable version (defined below) $[10,11]$, whereas the nonprojectable case still remains elusive. Second, the ultraviolet structure of the theory, essential to establish its consistency, is not known beyond tree level. Partial results include the study of renormalization group (RG) flow in a simplified model obtained from the projectable theory by a conformal reduction of the metric [12] and the calculation of the contributions of matter loops to the RG running of the couplings [13].

In this Letter we make the next step in this direction and compute the complete $\beta$ functions in the pure projectable Hoŕava gravity in $2+1$ dimensions, for the first time fully taking into account the gravitational degrees of freedom (d.o.f.). It is worth noting that, unlike $(2+1)$-dimensional GR, the theory possesses a local propagating scalar mode [14]. We find that there is a region in parameter space where the projectable theory is asymptotically free. This implies that projectable Hořava gravity is a perturbatively complete theory of quantum gravity in $2+1$ dimensions. 
Horava gravity (projectable case).-The action of Hořava gravity in $d+1$ spacetime dimensions is constructed by considering theories invariant under the diffeomorphisms compatible with a preferred foliation of space-time

$$
t \mapsto t^{\prime}(t), \quad x^{i} \mapsto x^{\prime i}(x, t) .
$$

This suggests to decompose the metric in the ArnowittDeser-Misner (ADM) form (we perform our calculation in Euclidean signature)

$$
d s^{2}=N^{2} d t^{2}+\gamma_{i j}\left(d x^{i}+N^{i} d t\right)\left(d x^{j}+N^{j} d t\right),
$$

$i, j=1,2, \ldots, d$. Here, $N$ is the lapse function, $N^{i}$ the shift vector, and $\gamma_{i j}$ the metric on the spatial codimension-one hypersurfaces which foliate the spacetime. The gravitational action with only marginal and relevant couplings with respect to the Lifshitz scaling (1) reads,

$$
S=\frac{1}{2 G} \int d t d^{d} x N \sqrt{\gamma}\left(K_{i j} K^{i j}-\lambda K^{2}+\mathcal{V}\right),
$$

where $\lambda$ and $G$ are coupling constants while $K_{i j}$ is the extrinsic curvature of the foliation

$$
K_{i j}=\frac{1}{2 N}\left(\partial_{t} \gamma_{i j}-\nabla_{i} N_{j}-\nabla_{j} N_{i}\right),
$$

with $\nabla_{i}$ being the covariant derivative compatible with $\gamma_{i j}$ and $K \equiv \gamma^{i j} K_{i j}$. The potential $\mathcal{V}$ includes all possible terms constructed out of the covariant derivative $\nabla_{i}$, the spatial Riemann tensor $R_{i j k l}$ and the acceleration vector $a_{i}=\partial_{i} \log N$, that under Eq. (1) have scaling dimensions $2 d$ or less. The action (4) is then power-counting renormalizable [4]. The case of GR would correspond to $\lambda=1$ and $\mathcal{V}=2 \Lambda-R$, where $\Lambda$ is the cosmological constant and $R$ is the intrinsic curvature of the $d$-dimensional slices.

The symmetry (2) can be satisfied if $N$ depends only on time $\left(a_{i}=0\right)$, which defines the projectable version of Hořava gravity. The action for this proposal was derived in Refs. [15,16] and it was shown to be renormalizable beyond power counting in Refs. [10,11]. This proposal is the focus of this work. We also concentrate only on the case $d=2$. The previous restrictions reduce the number of terms in $\mathcal{V}$ drastically, while keeping nontrivial (gravitational) local d.o.f. [14]. In the projectable case, the lapse function $N$ does not affect the local dynamics. By fixing the time coordinate it can be set to $N=1$ in perturbation theory, which we will assume in what follows. The remaining gauge invariance consists of time-dependent spatial diffeomorphisms. An important caveat is that this theory is not phenomenologically viable in $3+1$ dimensions [17]. Nevertheless, one can view it as a toy model for quantum gravity with dynamical d.o.f.
Quantizing projectable Hořava gravity in $2+1$.-For $d=2$ the potential term of projectable Hořava gravity has the form,

$$
\mathcal{V}=2 \Lambda+\mu R^{2} .
$$

The term linear in $R$ is not present, as it is a total derivative in $2+1$ dimensions. Our aim is to perform a one-loop calculation and to analyze the running of the marginal couplings that define the theory. We do this by using the background field technique [18], where the renormalization of the coupling constants is captured by the contributions to operators of the background fields, coming from integrating out the quantum fluctuations around the background. The anomalous dimension of the cosmological constant was already computed in Ref. [19]. We focus on the case $\Lambda=0$ and study the renormalization group (RG) running of the three marginal couplings $\{G, \lambda, \mu\}$ governing the UV dynamics of the theory. Only two combinations $\{\mathcal{G} \equiv$ $G / \sqrt{\mu}, \lambda\}$ are physical in the sense that their $\beta$ functions do not depend on the choice of the gauge. Conversely, all gauge-invariant quantities depend only on these combinations. This is a consequence of the fact that a change of gauge shifts the off-shell effective action by a contribution that vanishes on shell $[20,21]$. The equations of motion following from Eqs. (4) and (6) imply the global Hamiltonian constraint,

$$
\mathcal{H} \equiv \int d^{2} x \sqrt{\gamma}\left[K_{i j} K^{i j}-\lambda K^{2}-\mu R^{2}\right]=0 .
$$

This is also a unique combination of marginal gaugeinvariant operators vanishing on shell. Thus, the 1-loop effective action $\Gamma\left[\gamma_{i j}, N_{i}\right]$ is defined up to the transformations,

$$
\Gamma \rightarrow \Gamma+\varepsilon \int d t d^{2} x \sqrt{\gamma}\left[K_{i j} K^{i j}-\lambda K^{2}-\mu R^{2}\right],
$$

with arbitrary constant $\varepsilon$. Such a shift corresponds to the following transformation of couplings $\delta G=-2 G^{2} \varepsilon$, $\delta \lambda=0$ and $\delta \mu=-4 G \mu \varepsilon$. Only the $\beta$ functions of the essential couplings $\lambda$ and $\mathcal{G}$, which are invariant under these transformations, have physical meaning. Here $\lambda$ measures the deviation from a relativistic invariant kinetic term, while $\mathcal{G}$ controls the strength of gravitational interactions.

In order to compute $\Gamma\left[\gamma_{i j}, N_{i}\right]$, we expand the fields around an arbitrary background configuration

$$
\gamma_{i j}=\bar{\gamma}_{i j}+h_{i j}, \quad N_{i}=0+n_{i},
$$

where we have set $\bar{N}_{i}=0$. This choice simplifies the calculation without losing information about the $\beta$ functions, since $\bar{N}_{i}$ only enters via $\bar{K}_{i j}$. Expanding the bare action (4) up to second order in the quantum fluctuations 
yields the quadratic action $S_{2}$, which is sufficient to capture the one-loop contributions. For notational simplicity, we omit the bars over background quantities in what follows.

We add to $S_{2}$ a gauge fixing term implementing the regular gauge proposed in Ref. [10],

$$
S_{\mathrm{gf}}=\frac{\sigma}{2 G} \int d t d^{2} x \sqrt{\gamma} F_{i} \frac{-1}{\gamma_{i j} \Delta+\xi \nabla_{i} \nabla_{j}} F_{j},
$$

with the gauge fixing condition $F_{i}$ given by

$$
F_{i}=\partial_{t} n_{i}-\frac{1}{2 \sigma}\left(\gamma_{i j} \Delta+\xi \nabla_{i} \nabla_{j}\right)\left(\nabla^{k} h_{k}^{j}-\lambda \nabla^{j} h\right)
$$

This gauge fixing leads to regular propagators featuring a uniform scaling structure, which is a key element in the proof of the renormalizability of the theory [10,22]. Moreover, this choice of $F_{i}$ removes the mixing between $h_{i j}$ and $n_{i}$ in the quadratic part of the action. All the terms in $S_{\text {gf }}$ are local-except for one, which, however, can be written in a local form by "integrating in" the extra auxiliary field $\pi^{i}[10]$,

$$
\begin{aligned}
& \frac{\sigma}{2 G} \int d t d^{2} x \sqrt{\gamma} \partial_{t} n_{i} \frac{-1}{\gamma_{i j} \Delta+\xi \nabla_{i} \nabla_{j}} \partial_{t} n_{j} \\
& \mapsto \frac{1}{2 G} \int d t d^{2} x \sqrt{\gamma}\left(\frac{-1}{2 \sigma} \pi^{i}\left(\gamma_{i j} \Delta+\xi \nabla_{i} \nabla_{j}\right) \pi^{j}-i \pi^{i} \partial_{t} n_{i}\right) .
\end{aligned}
$$

Finally, we need to include the corresponding ghost action, which is constructed in the standard way from the gauge fixing condition $F_{i}[10,19]$,

$$
\begin{aligned}
S_{\mathrm{gh}}= & -\int d t d^{2} x \sqrt{\gamma} \bar{c}^{i}\left(\partial_{t}\left(\gamma_{i j} \partial_{t} c^{j}\right)-\frac{1}{2 \sigma} \Delta^{2}\left(\gamma_{i j} c^{j}\right)\right. \\
& -\frac{1}{2 \sigma} \Delta \nabla_{k} \nabla_{i} c^{k}+\frac{\lambda}{\sigma} \Delta \nabla_{i} \nabla_{j} c^{j}-\frac{\xi}{2 \sigma}\left(\nabla_{i} \nabla_{j} \Delta c^{j}\right. \\
& \left.\left.+\nabla_{i} \nabla_{j} \nabla_{k} \nabla^{j} c^{k}-2 \lambda \nabla_{i} \Delta \nabla_{j} c^{j}\right)\right) .
\end{aligned}
$$

Given the total action for the quantum fluctuations $S_{\text {tot }}=S_{2}+S_{\mathrm{gf}}+S_{\mathrm{gh}}$, we use perturbation theory around a background metric $\gamma_{i j}$ which is close to flat space $\delta_{i j}$,

$$
\gamma_{i j}=\delta_{i j}+H_{i j}
$$

Because of the invariance of the effective action with respect to the background diffeomorphisms, it is sufficient to focus on the renormalization of the terms quadratic in $H_{i j}$. The bare action quadratic in $H_{i j}$ reads

$$
\begin{aligned}
S_{H}= & \frac{1}{2 G} \int d t d^{2} x\left\{\frac{1}{4}\left(\dot{H}_{i j} \dot{H}^{i j}-\lambda \dot{H} \dot{H}\right)\right. \\
& \left.-\mu \partial_{b} \partial_{a} H^{a b}\left(2 \Delta H-\partial_{j} \partial_{i} H^{i j}\right)+\mu \Delta H \Delta H+O\left(H^{3}\right)\right\} .
\end{aligned}
$$

The $\beta$ functions are found by studying how the two-point functions of $H_{i j}$, following from Eq. (15), are renormalized after integrating out the quantum fluctuations. The renormalization of $G$ is then extracted from $\dot{H}_{i j} \dot{H}^{i j}$, while the one of $\lambda$ comes from $\dot{H} \dot{H}$. For the renormalization of $\mu$ we can use any of the three other structures in $S_{H}$.

The background independent part of the second order action defines the propagators for $h_{i j}, n_{i}, \pi_{i}, \bar{c}^{i}$, and $c^{i}$. The latter are particularly simple in the gauge

$$
\sigma=\frac{1-2 \lambda}{8 \mu(1-\lambda)}, \quad \xi=-\frac{1-2 \lambda}{2(1-\lambda)}
$$

where they are all proportional to the propagator of the physical scalar mode,

$$
\mathcal{P}_{s}(\omega, p)=\left[\omega^{2}+4 \mu \frac{1-\lambda}{1-2 \lambda} p^{4}\right]^{-1} .
$$

The vertices required for the one-loop calculation can be found by expanding $S_{\text {tot }}$ up to second order in the background field $H_{i j}$. The diagrams which give rise to logarithmic divergences are shown in Fig. 1.

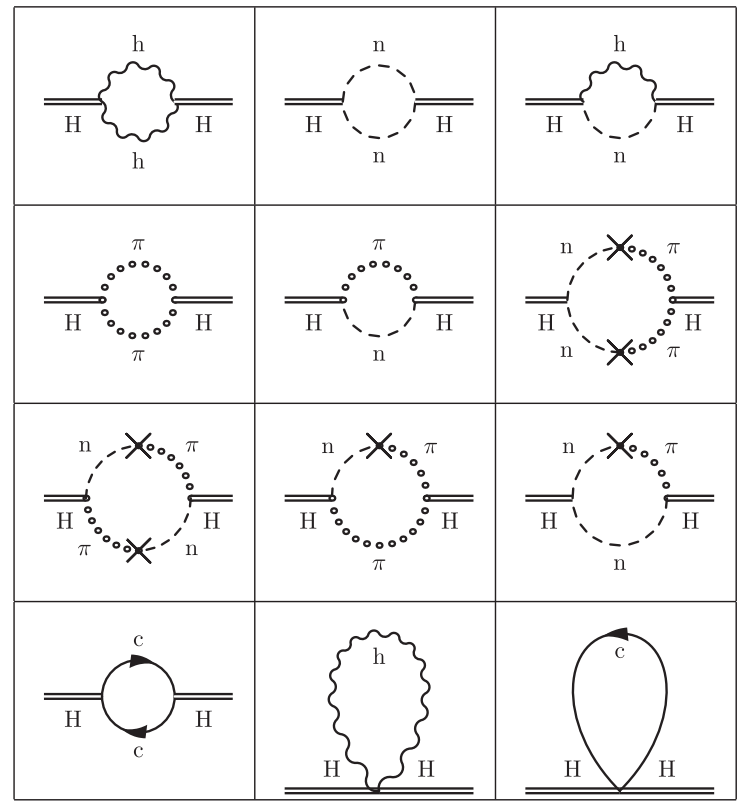

FIG. 1. Feynman diagrams (bubbles and fishes) for the two point function of $H_{i j}$. The cross represents the mixed propagator $\left\langle n^{i} \pi^{j}\right\rangle$. 
We stress that the possibility to study only the renormalization of terms quadratic in $H_{i j}$ essentially relies on our procedure where we start from the action for perturbations $S_{\text {tot }}$ invariant under the background gauge transformations, including the gauge fixing and ghost terms. Only after that we expand the background according to Eq. (14). Had we started by first expanding the metric around flat spacetime and then fixing a (noncovariant) gauge, we would have to compute also the renormalization of 3- and 4-point vertices to factor out the (gauge-dependent) wave function renormalization.

Calculation of diagrams. - Although the number of diagrams in Fig. 1 is not so large, the different vertices contain multiple terms which make computations rather involved and lengthy. To handle this complexity we use the Mathematica package $x A c t$ [23] to manipulate the algebraic expressions and FORM [24] to reduce the output of the diagrams. The computation is simplified by considering the renormalization of $\{G, \lambda\}$ and of $\mu$ separately. This can be extracted by evaluating the quadratic part of the effective action $\Gamma\left[H_{i j}\right]$ on time- or space-dependent backgrounds which correspond, respectively, to diagrams with vanishing spatial momenta or frequency in external legs. Thus, for $\{G, \lambda\}$ renormalization we first focus on computing the contributions carrying only external frequency $\Omega$ at vanishing external momentum $P_{i}$. Then we do the opposite for $\mu$ renormalization and compute the logarithmically divergent diagrams carrying only external momentum at vanishing $\Omega$.

The prototypical loop integral over internal momentum and frequency has the form,

$$
\int \frac{d \omega d^{2} q}{(2 \pi)^{3}} \omega^{2 a} q^{2 b} \prod_{I} \mathcal{P}_{s}\left(\omega+\Omega_{I}, q+P_{I}\right),
$$

with constant exponents $a, b$. Here $\Omega_{I}$ and $P_{I}$ are the relevant external frequencies and two-momenta. Since we are interested in the logarithmic divergences, we retain only the contributions proportional to $\Omega^{2}$ or $P^{4}$, which renormalize the terms shown in (15). For this, we Taylor expand the integrand of Eq. (18) up to the desired order in external frequency or momentum, such that the final integrands all acquire the general form

$$
\mathcal{I}[a, b, A]=\omega^{2 a} q^{2 b}\left[\mathcal{P}_{s}(\omega, q)\right]^{A},
$$

with $A$ being a constant power. We regularize the UV divergences, which appear in the integration of Eq. (19), by using the Schwinger integral representation for the propagator. To this end, we introduce an auxiliary "proper time" parameter $s$ and rewrite Eq. (19) as

$$
\mathcal{I}[a, b, A]=\omega^{2 a} q^{2 b} \int_{0}^{\infty} \frac{d s s^{A-1}}{\Gamma(A)} e^{-s\left[\mathcal{P}_{s}(\omega, q)\right]^{-1}} .
$$

The integral over frequency and momentum in Eq. (18) can then be expressed in terms of the $\Gamma$ function. In the
Schwinger integral representation UV divergences appear in the limit $s \rightarrow 0$, with logarithmic divergences corresponding to $\int d(\log s)$.

One-loop $\beta$ functions and asymptotic freedom.-In the Wilsonian picture the quantum corrections are interpreted as the result of integrating out virtual modes with momenta between a certain UV cutoff $\Lambda_{\mathrm{UV}}$ and the subtraction point $k_{*}$. This leads to the following identification in the divergent part of the effective action,

$$
\int d(\log s) \mapsto \log \left(\frac{\Lambda_{\mathrm{UV}}^{4}}{k_{*}^{4}}\right),
$$

where we have taken into account that the parameter $s$ has scaling dimension 4 . The RG flow originates from the sensitivity of the couplings to a change in $k_{*}$, see, e.g., Ref. [25]. We find the following $\beta$ functions for the physical couplings:

$\beta_{\lambda} \equiv k_{*} \frac{d \lambda}{d k_{*}}=\frac{15-14 \lambda}{64 \pi} \sqrt{\frac{1-2 \lambda}{1-\lambda}} \mathcal{G}$,

$\beta_{\mathcal{G}} \equiv k_{*} \frac{d \mathcal{G}}{d k_{*}}=-\frac{\left(16-33 \lambda+18 \lambda^{2}\right)}{64 \pi(1-\lambda)^{2}} \sqrt{\frac{1-\lambda}{1-2 \lambda}} \mathcal{G}^{2}$.

We have checked this result by performing independent calculations in several gauges other than Eq. (16). Namely, we have considered the gauge (10) with $\xi=0$ [and with $\sigma$ as in (16)] and, outside of the family (10), the conformal gauge $h_{i j}=e^{2 \phi} \gamma_{i j}$ which is possible in two spatial dimensions [26]. Furthermore, the (gauge-dependent) $\beta$ function for the coupling $\mu$ can be extracted from the results of Ref. [19] and it agrees with our results when evaluated in the same gauge.

The structure of the RG flow in the regions required by unitarity [10], $\{\lambda<1 / 2\} \cup\{\lambda>1\}$, is shown in Fig. 2. The theory possesses two UV fixed points:

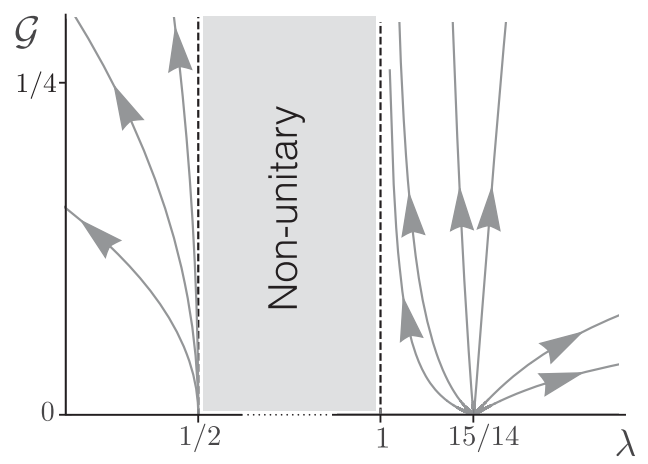

FIG. 2. RG flow of the couplings in $(2+1)$-dimensional Horava gravity. The arrows show the direction of the flow towards the infrared. 


$$
(\lambda, \mathcal{G})=\left(\frac{1}{2}, 0\right) \quad \text { and } \quad(\lambda, \mathcal{G})=\left(\frac{15}{14}, 0\right) .
$$

The first fixed point is located at the boundary of the allowed region. The limit $\lambda \rightarrow 1 / 2$, taken at fixed value of $\mathcal{G}$, is strongly coupled, as it is clear from the divergence in Eq. (22b). However, the RG flow $\lambda \rightarrow 1 / 2$ is accompanied by the vanishing of $\mathcal{G}$. The structure of $\beta_{\mathcal{G}}$ in Eq. (22b) suggests that the actual expansion parameter in this limit is [27] $\tilde{\mathcal{G}}=\mathcal{G} / \sqrt{1-2 \lambda}$, with the $\beta$ function

$$
\beta_{\tilde{\mathcal{G}}}=-\frac{(1-2 \lambda)^{2}}{64 \pi(1-\lambda)^{3 / 2}} \tilde{\mathcal{G}}^{2} .
$$

This $\beta$ function vanishes at $\lambda \rightarrow 1 / 2$, so that $\tilde{\mathcal{G}}$ freezes at a constant value in the UV. In other words, at the one-loop level there is a family of UV fixed points parametrized by the asymptotic value of $\tilde{\mathcal{G}}$. The status of this fixed-point family can be clarified only by taking into account contributions from higher order and matter loops.

Remarkably, the second UV fixed point in Eq. (23) is regular and asymptotically free. In the infrared (IR), the RG trajectories either go to $\lambda \rightarrow+\infty, \mathcal{G} \rightarrow+\infty$, or to $\lambda \rightarrow 1^{+}$, $\mathcal{G} \rightarrow+\infty$. The latter behavior is intriguing as it naively corresponds to the relativistic limit of the theory. However, to decide whether the theory really flows or not to GR requires a nonperturbative analysis as in the IR the system enters into the strong-coupling regime, which is typical for asymptotically free theories [28].

It is worth comparing our results to those of Ref. [12], where the gravitational d.o.f. were truncated to the conformal mode. While the family of fixed points at $\lambda \rightarrow 1 / 2$ is present also in the truncated model, the fixed point at $\lambda=15 / 14$ exists only in the full theory. A more important difference is that the asymptotic freedom of the gravitational coupling reported in Ref. [12] occurrs at negative values of $G$ - the choice required by unitarity of the truncated model at $\lambda>1 / 2$. On the other hand, the full theory is both unitary and asymptotically free at positive $G$ and $\lambda>1$.

Conclusions.-The main result presented in this Letter are the $\beta$ functions (22) for the essential coupling constants of projectable Hořava gravity in $2+1$ dimensions. Their associated flow is shown in Fig. 2. The RG flow possesses an asymptotically free fixed point in the UV, which establishes this model as a $2+1$ dimensional perturbatively UV-complete theory with nontrivial propagating gravitational d.o.f. This not only makes it a theory of quantum gravity in $2+1$ dimensions but also a suitable toy model to address fundamental aspects of quantum gravity in the more realistic $(3+1)$-dimensional case.

We are grateful to Renate Loll and Frank Saueressig for discussions. M. H-V. is grateful to Jos Vermaseren for his help with FORM during early stages of this work. C. S. is grateful for the hospitality of the CERN theory division.
This work was supported by the RFBR Grant No. 17-0200651 (A. B. and S. S.), the Tomsk State University Competitiveness Improvement Program (A. B.), the Tomalla Foundation (M. H.-V.) and the Swiss National Science Foundation (S. S.).

*mario.herrerovalea@epfl.ch

[1] K. S. Stelle, Phys. Rev. D 16, 953 (1977).

[2] E. S. Fradkin and A. A. Tseytlin, Phys. Lett. B 104, 377 (1981).

[3] I. G. Avramidi and A. O. Barvinsky, Phys. Lett. B 159, 269 (1985).

[4] P. Horava, Phys. Rev. D 79, 084008 (2009).

[5] S. Liberati, Classical Quantum Gravity 30, 133001 (2013).

[6] D. Blas, O. Pujolas, and S. Sibiryakov, Phys. Rev. Lett. 104, 181302 (2010).

[7] D. Blas and E. Lim, Int. J. Mod. Phys. D 23, 1443009 (2014).

[8] S. Janiszewski and A. Karch, J. High Energy Phys. 02 (2013) 123.

[9] T. Griffin, P. Horava, and C. M. Melby-Thompson, Phys. Rev. Lett. 110, 081602 (2013).

[10] A. O. Barvinsky, D. Blas, M. Herrero-Valea, S. M. Sibiryakov, and C. F. Steinwachs, Phys. Rev. D 93, 064022 (2016).

[11] A. O. Barvinsky, D. Blas, M. Herrero-Valea, S. M. Sibiryakov, and C. F. Steinwachs, arXiv:1705.03480.

[12] D. Benedetti and F. Guarnieri, J. High Energy Phys. 03 (2014) 078.

[13] G. D’Odorico, F. Saueressig, and M. Schutten, Phys. Rev. Lett. 113, 171101 (2014).

[14] T. P. Sotiriou, M. Visser, and S. Weinfurtner, Phys. Rev. D 83, 124021 (2011).

[15] T. P. Sotiriou, M. Visser, and S. Weinfurtner, Phys. Rev. Lett. 102, 251601 (2009).

[16] T. P. Sotiriou, M. Visser, and S. Weinfurtner, J. High Energy Phys. 10 (2009) 033.

[17] D. Blas, O. Pujolas, and S. Sibiryakov, J. High Energy Phys. 04 (2011) 018.

[18] L. F. Abbott, Acta Phys. Pol. B 13, 33 (1982).

[19] T. Griffin, K. T. Grosvenor, C. M. Melby-Thompson, and Z. Yan, J. High Energy Phys. 06 (2017) 004.

[20] B. S. DeWitt, Phys. Rev. 162, 1195 (1967).

[21] R. E. Kallosh, Nucl. Phys. B78, 293 (1974).

[22] D. Anselmi, Ann. Phys. (Amsterdam) 324, 874 (2009).

[23] D. Brizuela, J. M. Martin-Garcia, and G. A. Mena Marugan, Gen. Relativ. Gravit. 41, 2415 (2009).

[24] T. Ueda and J. Vermaseren, J. Phys. Conf. Ser. 523, 012047 (2014).

[25] S.-B. Liao, Phys. Rev. D 53, 2020 (1996).

[26] A. O. Barvinsky, D. Blas, M. Herrero-Valea, S. M. Sibiryakov, and C. F. Steinwachs (to be published).

[27] The analysis of the interaction terms in the action confirms that this combination controls the coupling strength when $\lambda \rightarrow 1 / 2$.

[28] If this behavior persists in higher dimensions, the strong coupling in the IR will be naturally cut off by relevant deformations, which do not exist in $2+1$ dimensions, cf. Eq. (6). 